\title{
Community services as punishment and safety road: Questions and prospects
}

\section{Pena de trabajos en beneficio de la comunidad y seguridad vial: Cuestiones y perspectivas}

\author{
Vidales Rodríguez Caty ${ }^{1}$
}

Published February 2011

(C) Etrasa, 2011

\begin{abstract}
The Criminal Code Reform with regard to road safety enacted by the Organic Law $15 / 2007$, November 30, has increased noticeably the criminal convictions imposing community services as punishment. However, these convictions have not involved the appropriate increase of job offers for providing those community services. The high risk of failure to comply with community services as punishment has forced to formulate new proposals. In that respect, the content of the punishment has changed. So, this punishment can be served participating in workshops or attending at a driver education program. Despite the favorable effects of re-socialization of this measure, however, overlapping with other training activities of a similar entity made a clear demarcation necessary.
\end{abstract}

1.- Vidales Rodríguez, Caty.

Profesora titular de Derecho Penal

Universitat Jaume I.
Keywords. Punishment, Community Services, Road Safety, Driver Education Program.

Resumen. La reforma del Código penal en materia de seguridad vial que llevó a cabo la Ley Orgánica $15 / 2007$, de 30 de noviembre, ha supuesto un notable incremento de condenas en las que se impone la pena de trabajos en beneficio de la comunidad, sin que se haya producido un correlativo aumento de la oferta de los puestos de trabajo donde realizar la prestación. El elevado riesgo de incumplimiento de tal sanción ha obligado a formular nuevas propuestas. En este sentido, se ha modificado el contenido de esta pena de forma que pueda cumplirse a través de la participación del penado en talleres o programas de educación vial. Pese a los efectos claramente resocializadores de esta medida puede, no obstante, solaparse con otro tipo de actividades formativas de análoga entidad por lo que procederá un claro deslinde.

Palabras Clave. Penas, Trabajos en beneficio de la comunidad, Seguridad Vial, Programas de educación vial. 


\section{Sumario:}

I.- Introducción.

II.- La participación en actividades formativas como modalidad de cumplimiento de la pena de trabajos en beneficio de la comunidad.

a) Los talleres de educación vial y los cursos de reeducación y sensibilización vial.

b) Los talleres de educación vial y los programas formativos de educación vial

III.- A modo de conclusión.

IV.- Bibliografía.

\section{Introducción}

La pena de trabajos en beneficio de la comunidad aparece, por primera vez en nuestra legislación penal, con ocasión de la aprobación del Código penal de 1995 en el que se considera una pena privativa de derechos. Bien es cierto que, a pesar de los efectos beneficiosos que puede reconocérsele y que la doctrina se ha ocupado de poner de manifiesto [1], se trata de una aparición tímida y casi residual al estar únicamente prevista como pena sustitutiva de los arrestos de fin de semana o en caso de impago de la multa.

La reforma operada en el texto punitivo mediante la Ley Orgánica 11/2003, de 29 de septiembre, supuso que, por primera vez, la realización de determinados comportamientos relacionados con la violencia de género se conminara con este tipo de sanción, aunque de forma alternativa al requerirse el consentimiento del penado. Habría que esperar, no obstante, hasta la aprobación de la Ley Orgánica 15/2003, de 25 de noviembre, para que el número de conductas cuya consecuencia jurídica pudiera ser la pena que se comenta experimentara un notable incremento debido, en buena parte, a la desaparición del arresto de fin de semana del catálogo de sanciones penales. Ahora bien, esta reforma, lejos de limitarse a un mero aumento cuantitativo, introduce, asimismo, un importante cambio en su contenido. En efecto, de un lado, se potencian los aspectos resocializadores y reeducadores de la pena, al especificar que los trabajos a prestar puedan consistir en labores de reparación de los daños causados o de apoyo o asistencia a las víctimas y, de otro, se incorpora por primera vez al texto punitivo el régimen jurídico de su incumplimiento.

Por lo que respecta a los entonces denominados delitos contra la seguridad del tráfico, pese a tratarse de un ámbito de la delincuencia especialmente propicio para la realización de trabajos en beneficio de la comunidad, únicamente estaba prevista $\mathrm{y}$, dicho sea de paso, mediante el empleo de una fórmula legislativa bastante confusa, en relación con el comportamiento descrito en el artículo 379 del Código penal; esto es, la conducción bajo la influencia de drogas tóxicas, estupefacientes, sustancias psicotrópicas o de bebidas alcohólicas.

La sustancial modificación que en este ámbito lleva a cabo la Ley Orgánica 15/2007, de 30 de noviembre [2], ha concedido a la pena de trabajos en beneficio de la comunidad un protagonismo hasta entonces desconocido. Así es por cuanto que, como pena principal y alternativa a la privación de libertad, acompaña a la sanción pecuniaria en relación a las dos modalidades delictivas que contemplan los artículos 379 (conducción a velocidad superior a la permitida y conducción bajo la influencia de drogas tóxicas, estupefacientes, sustancias psicotrópicas o de bebidas alcohólicas), 384 (quebrantamiento de condena o de resolución administrativa y conducción sin permiso o licencia) y 385 (creación de grave riesgo para la circulación). Además de estos supuestos, conviene tener presente que puede operar en sustitución de la pena de prisión en aquellos casos en los que concurran, claro está, los requisitos establecidos a tal efecto en el artículo 88 del Código penal [3].

Tenemos, por tanto, que la pena de trabajos en beneficio de la comunidad puede ser aplicada en relación con la mayor parte de los comportamientos típicos que engloba esta categoría delictiva. De ahí que, las previsiones legislativas se hayan visto desbordadas, debido fundamentalmente a que el elevado número de condenas en las que se impone esta pena no se ha visto acompañado por un aumento equivalente en la oferta de puestos de trabajo para su realización. La 
seriedad del problema no se esconde en la Memoria del Fiscal General del Estado que, en 2008, tilda de gravísimo el incumplimiento de esta sanción por carencia de medios [4] y, no es para menos, si tenemos en cuenta que el número de condenas impuestas durante ese año por este tipo de delitos gira en torno a las 30.000 de las cuales, aproximadamente un 80 $\%$ estaban pendientes de ser ejecutadas existiendo, respecto de ellas, un alto riesgo de prescripción [5]. La situación, sin embargo, parece cambiar en 2009 porque, aunque se produce un incremento considerable de sentencias condenatorias -que supera con creces las 120.000-, las medidas que se han ido adoptando permiten augurar una mejora en el nivel de cumplimiento de las mismas; razón por la que se hace gala de un claro optimismo [6].

\section{La participación en actividades for- mativas como modalidad de cumpli- miento de la pena de trabajos en be- neficio de la comunidad}

Al confesado propósito de contribuir a paliar la situación brevemente descrita obedece la reforma del Real Decreto 515/2005, de 6 de mayo, que establece las circunstancias de ejecución de las penas de trabajos en beneficio de la comunidad y de localización permanente, de determinadas medidas de seguridad, así como de la suspensión de la ejecución de las penas privativas de libertad. Dicha modificación se ha llevado a cabo por medio del Real Decreto 1849/2009, de 4 de diciembre. Entre las novedades que introduce, cabe destacar, por el interés que reviste en el ámbito que nos ocupa, aquella que afecta al apartado 4 del artículo 6, en cuya virtud se establece la posibilidad de que la pena de trabajos en beneficio de la comunidad pueda cumplirse mediante la realización de talleres de actividades en materia de seguridad vial. Dichos talleres, a tenor de lo dispuesto en el citado precepto, han de constar de una fase formativa y de otra de realización de actividades de utilidad pública.

Por su parte, la Ley Orgánica 5/2010, de 22 de junio, generaliza esta posibilidad sin limitarla ya, como inicialmente estaba previsto, a los delitos contra la seguridad vial. A tal fin modifica el artículo 49 del Código penal lo que, sin duda, resulta más respetuoso con las exigencias derivadas del principio de legalidad.

De obligada referencia en este contexto es el Taller de actividades para el cumplimiento de trabajos en beneficio de la comunidad en delitos con la seguridad vial más conocido como TASEVAL y que, con carácter general, persigue dar cumplimiento a la pena de trabajos en beneficio de la comunidad a través de una serie de actividades formativas y actividades de utilidad pública directamente relacionadas con el tráfico para, de este modo, reducir la tasa de reincidencia. Está pensado para condenas a trabajos en beneficio de la comunidad de hasta 40 jornadas de trabajo [7] y, como es preceptivo, se articula en dos fases. En la primera, formativa y con una duración de 20 horas, se trabajan determinados contenidos relacionados con esta problemática y se presta especial atención a las principales causas de los accidentes (velocidad, consumo de alcohol y drogas, conducción temeraria o distracciones); en la segunda, cuya duración vendrá determinada por la concreta pena a cumplir, se realizan las correspondientes actividades de utilidad pública dirigidas a prevenir accidentes de tráfico, entre las que pueden mencionarse, a modo de ejemplo, la colaboración en campañas de prevención, la observación y registro de zonas en las que se constata un elevado número de accidentes, la detección de señales de tráfico deterioradas o mal situadas, la elaboración de un listado de calzadas con el pavimento en mal estado o la observación de pasos de peatones mal señalizados y/o con escasa visibilidad [8].

Ni que decir tiene que la participación de los condenados en este tipo de talleres, supone una importante potenciación de la función resocializadora y reeducadora que las penas están llamadas a cumplir y más, como es el caso, cuando se vincula el delito cometido con la actividad a realizar. Por ello, en mi opinión, estas iniciativas merecen una valoración positiva. Sin embargo, no puede decirse que se trate de una opción exenta de reparos pues, como a continuación veremos, puede producirse un solapamiento con otras acciones que ya existen y que tienen un contenido análogo, por lo que se hace necesario efectuar una clara delimitación entre unas y otras. 


\section{a.- Los talleres de educación vial y los cursos de reeducación y sensibiliza- ción vial:}

Como es sabido, la realización de estos cursos permite, según el caso, la recuperación parcial de puntos o la obtención de un nuevo permiso o licencia de conducción cuya pérdida ha sido consecuencia de la realización de conductas constitutivas de infracción administrativa. La necesidad de diferenciarlos, respecto de la nueva modalidad de cumplimiento de la pena de trabajos en beneficio de la comunidad que ahora se introduce, es evidente si tenemos en cuenta que a ellos parece aludir el propio Real Decreto $1849 / 2009$, al poner de manifiesto que "la modificación atiende a la necesidad de utilizar unos recursos ya existentes de utilidad pública de similar naturaleza en su finalidad reparadora al delito cometido por el penado". Ahora bien, como se advirtiera en el Informe del Consejo General del Poder Judicial, en relación con el borrador de Decreto que se sometió a su consideración, esta equiparación plantea serios problemas pues se trata, e innecesario tendría que ser recordarlo, de dos actividades que, aunque relativas a idéntica problemática, difieren en cuanto a su naturaleza, fundamento, régimen jurídico y, por todo ello, también son distintas las consecuencias que pueden derivar de su eventual incumplimiento.

Así las cosas, no debe extrañar que en la Instrucción a la que se ha hecho referencia, se establezca que la participación en el referido taller, "es independiente de otras posibles acciones formativas de seguridad vial que el/la penado/a pueda o deba hacer; como por ejemplo, el curso de sensibilización y reeducación para la recuperación de los puntos del carné de conducir", para enfatizar a continuación que "no existe ningún tipo de relación ni de posible convalidación entre TASEVAL y cualquier otro curso de seguridad vial que pueda realizar el/la penado/a". Tenemos, pues, que la realización de los cursos de reeducación y sensibilización vial no exime del cumplimiento -bajo esta modalidad- de la pena impuesta.

Desde luego, nada hay que objetar al respecto de tan concluyente afirmación cuando la pérdida de la autorización para conducir obedece a la realización de conductas sancionadas administrativamente. Mas, quizás, esta conclusión debiera matizarse en relación al supuesto que contempla la disposición adicional decimotercera incorporada a la Ley sobre tráfico, circulación de vehículos a motor y seguridad vial de 1990 por medio de la Ley 17/2005, de 19 de julio, que regula el permiso y la licencia de conducción por puntos. En atención a lo que allí se establece, quienes hayan sido condenados por sentencia firme con la privación del derecho a conducir un vehículo a motor o ciclomotor deberán acreditar el haber superado con aprovechamiento el curso de reeducación y sensibilización vial al que se refiere el apartado séptimo del artículo 63 de dicha norma, como condición ineludible para volver a conducir.

En este sentido, no puede desconocerse que la mayor parte de los comportamientos que se consideran atentatorios contra la seguridad vial tienen prevista la pena de privación del derecho a conducir vehículos a motor y ciclomotores. Se exceptúan, por razones evidentes, la conducta descrita en el artículo 384 (quebrantamiento de condena o de resolución administrativa y conducción sin permiso o licencia) e, igualmente, la contenida en el artículo 385 (creación de grave riesgo para la circulación), al ser la única modalidad comisiva que no precisa el acto de conducción para su realización. Dejando al lado esta última modalidad, por su escasa virtualidad práctica, los datos que ofrece la Memoria de la Fiscalía General del Estado evidencian que el delito tipificado en el citado artículo 384 es de los que con mayor frecuencia se cometen, tan sólo superado por la conducción bajo la influencia de drogas tóxicas, estupefacientes, sustancias psicotrópicas o de bebidas alcohólicas [9]. Pero, para estos supuestos otro tipo de iniciativas, como la Campaña de universalización de la Educación Vial en los Centros Penitenciarios que está llevando a cabo la Secretaría General de Instituciones Penitenciarias [10], creo pueden ofrecer una respuesta más adecuada.

Volviendo a la declarada compatibilidad entre ambas actividades formativas, de mantenerse ésta, quien, como consecuencia de la comisión de un delito contra la seguridad vial, haya sido privado del derecho a conducir y haya participado en el referido taller debe superar, no obstante, el preceptivo curso como requisito ineludible para volver a ejercitar tal derecho. En estos casos, a diferencia del supuesto anterior, no estamos ya ante dos presupuestos distintos que, en 
consecuencia, deban merecer también un tratamiento diferenciado; sino que se trata de dos consecuencias jurídicas distintas en la forma, pero muy similares en cuanto al contenido [11], de un único hecho delictivo que, asimismo, comparten un mismo fundamento: la prevención de delitos contra la seguridad vial; por lo que la acumulación de las misma pudiera resultar excesiva.

\section{b.- Los talleres de educación vial y los programas formativos de educación vial:}

Similares inconvenientes plantea la nueva forma de cumplimiento de la pena de trabajos en beneficio de la comunidad en relación con las medidas que, con carácter facultativo, se pueden imponer en caso de suspensión o sustitución de la pena privativa de libertad, a las que alude el artículo 83.1.5 del Código penal. El citado precepto cita expresamente, entre otros, los programas formativos de educación vial; por lo que, a la vista de esta referencia, no resulta improcedente preguntarse si cabe diferenciar estos programas de los más novedosos talleres o si, por el contrario, se trata de términos equivalentes que se refieren a labores de parecida entidad.

$\mathrm{Al}$ respecto, es de tener en cuenta que el artículo 49 del Código penal, con la nueva redacción dada tras la reforma de 2010, se refiere tanto a programas como a talleres; no así, el Real Decreto 1849/2009, de 4 de diciembre, que únicamente aludía a estos últimos. Ello unido, a la ya mencionada posibilidad de conciliar la participación en TASEVAL con cualquier otra actividad formativa, hace pensar que se trata de dos cosas distintas. Un último argumento que parece avalar esta hipótesis es el diferente régimen que presentan.

En efecto, la participación en los tantas veces aludidos talleres ha de ir seguida inexorablemente de la realización no retribuida de una actividad de utilidad social, mientras que estarán exentos de tal obligación quienes hayan visto su condena privativa de libertad suspendida o sustituida. A lo que debe unírsele la preceptiva concurrencia del consentimiento del penado en caso de que se trate del cumplimiento de la pena de trabajos en beneficio de la comunidad. Además, conviene recordar que, en este último su- puesto, la supervisión y control corresponde al Juez de Vigilancia Penitenciaria y a los servicios sociales penitenciarios. Finalmente, hay que tener presente que, en caso de incumplimiento, pudiera derivar responsabilidad penal por delito de desobediencia (artículo $556 \mathrm{CP}$ ) o por delito de quebrantamiento de condena (artículo $468 \mathrm{CP}$ ), dependiendo de si se ha iniciado o no la ejecución de tal pena.

En cambio, cuando se trate de la obligación prevista en el artículo 83.1.5 , será el juez o tribunal sentenciador quien decida la conveniencia de someter al penado a tal deber y, asimismo, este será el órgano competente para realizar el seguimiento y control del cumplimiento de la medida. Por último, si se incumpliese la obligación impuesta, habría que distinguir en función de que se trate de un supuesto de suspensión o de sustitución de la pena. Así, en el primer caso, el juez o tribunal habría de decantarse por alguna de las posibilidades que le brinda el artículo 84.2, a saber, sustituir la regla de conducta por otra, prorrogar el plazo de suspensión o revocar la suspensión de la ejecución de la pena, si se tratase de incumplimientos reiterados; mientras que cuando se trata de la inobservancia de la pena sustitutiva, debería ejecutarse la pena de prisión en atención a lo dispuesto en el apartado segundo del artículo 88.

Con independencia del juicio que merezca este tratamiento tan dispar, lo cierto es que, al menos a nivel conceptual, las diferencias están claras. Como hemos visto, el único supuesto que puede plantear dudas es el que se refiere a la sustitución de la pena cuando el Juez, a la vista de la facultad que le confiere el artículo 88.1, decida imponer al penado alguna o algunas de las obligaciones o deberes previstos en el artículo 83, en concreto, la de participar en programas de educación vial. Si, en ese caso, la pena privativa de libertad se sustituyera por trabajos en beneficio de la comunidad y éstos consistieran en la participación en un taller de actividades en materia de seguridad vial habría que plantearse, la necesidad y conveniencia de la doble imposición. Cierto es, como ha quedado expuesto, que la Instrucción a la que se ha hecho referencia, no deja lugar a dudas; pero no menos cierto lo es el hecho de que, por imperativo legal, obligación y pena deben tener un contenido diferente. 
El problema se torna más complejo cuando a la pena privativa de libertad se suma la privación del derecho a conducir vehículos a motor y ciclomotores, lo que sucederá en un elevado porcentaje de casos. En estos supuestos, recordemos, es preceptiva la superación con aprovechamiento del curso de reeducación y sensibilización vial. De ahí que, podrían concurrir hasta tres actividades formativas distintas y, en principio, cumulativas: en primer lugar, el sometimiento a un programa de educación vial como consecuencia de la sustitución de la pena, si el juzgador estima conveniente imponer tal medida; en segundo lugar, la fase formativa del taller de actividades en materia de seguridad vial como modalidad de cumplimiento de la pena de trabajos en beneficio de la comunidad para el caso de que esta fuera la pena sustitutiva; y, por último, el sometimiento al mencionado curso para la recuperación del ejercicio del derecho a conducir.

\section{A modo de conclusión}

En lo que antecede se ha tratado de poner de manifiesto la incidencia que tiene la introducción de la nueva modalidad de cumplimiento de la pena de trabajos en beneficio de la comunidad en el ámbito de la delincuencia vial y su coexistencia, no siempre pacífica, con otras actividades formativas precedentes. De lo expuesto se deduce que en determinadas hipótesis se produce una imposición de deberes que pudiera resultar excesiva. Desconozco los efectos pedagógicos de este exceso pero, bien pudiera suceder que los importantes esfuerzos realizados tendentes a resocializar y reeducar a quien comete este tipo de infracciones fueran contraproducentes y que, de un lado, el carácter impositivo de tales medidas provoque una mayor rebeldía a la norma y, de otro, asiente la falsa creencia de su banalidad, con lo que la eficacia de los mismos se vería seriamente cuestionada.

En otro orden de consideraciones, hay que decir que la acumulación advertida aún resulta más perturbadora si se toma en consideración la absoluta escasez de acciones similares en los supuestos que, paradójicamente, pueden estar más necesitados de las mismas. Me refiero a los supuestos de reincidencia. En tales casos, es probable que el juzgador se decante -cuando haya alternativa- por la pena de prisión y, la existencia de una condena anterior, imposibilitará su suspensión, a salvo de la excepción que recoge el artículo 87 del Código penal; con lo que se impide la participación del penado en los programas a que se refiere el artículo 83.1.5. Los mismos motivos dificultarán la sustitución de la pena privativa de libertad; pero aunque así no fuera e, incluso, en el caso de que la pena de trabajos en beneficio de la comunidad se impusiera con carácter principal, la apreciación de la agravante y el consiguiente incremento punitivo que supone, impedirá la participación en el citado taller que, como se recordará, únicamente está previsto en relación con condenas que no excedan de 40 jornadas. En conclusión, estos sujetos únicamente verán cumplidas sus necesidades formativas a través de la realización con aprovechamiento de los cursos de reeducación y sensibilización vial en el caso de que la infracción cometida vaya acompañada de la privación del permiso del derecho a conducir y si, además, quieren volver a hacerlo.

En las consideraciones efectuadas no debe verse un motivo de discrepancia respecto de la introducción de la modalidad de cumplimiento de la pena que se comenta. Antes al contrario, creo que merece una buena acogida siempre, lógicamente, que no suponga la desnaturalización de los trabajos en beneficio de la comunidad y le haga perder su carácter punitivo. Por eso, es de lamentar que los reparos que pueda merecer enturbien la eficacia de una alternativa claramente resocializadora y reeducadora. Ante ese riesgo, tal vez habría que considerar la posibilidad de unificar todas las acciones formativas que tuvieran como presupuesto la comisión de un delito contra la seguridad vial y que, en consecuencia, estuviese diseñada en cuanto a duración, a contenido y a nivel de exigencia para proporcionar una respuesta homogénea y más adecuada a la grave problemática a la que se pretende hacer frente.

Además, y aunque ello reviste ciertamente una importancia menor, si así se hiciese quedaría despejada la duda en torno a si la reforma operada ha estado motivada por el firme convencimiento de las posibilidades resocializadoras que puede ofrecer la pena de trabajos en beneficio de la comunidad o, en cambio, obedece a la coyuntural necesidad de evitar un elevado índice de incumplimiento de la misma debido 
a la falta de puestos donde realizar las correspondientes labores [12].

De ser este último el objetivo prioritario, la modificación que la Ley Orgánica 5/2010 opera en los artículos 379 y 384 del Código penal conduce, en mi opinión, a resultados más satisfactorios ya que, en ambos casos, la pena de trabajos en beneficio de la comunidad deja de acompañar necesariamente a la sanción pecuniaria y pasa a ser, junto a la prisión y a la multa, una de las opciones por la que habrá de decantarse el juzgador.

Esta modificación sirve, asimismo, para atemperar el excesivo rigor punitivo de que hizo gala la reforma operada por medio de la ley 15/2007, de 30 de noviembre; de hecho, en el Preámbulo de la misma se alude al principio de proporcionalidad. Así se explica, igualmente, la potestad de atenuar la responsabilidad penal que confiere el nuevo artículo 385 ter. Cuestión distinta es si algunas de las conductas a que se refiere, lejos de merecer un reproche penal -ni siquiera de forma atenuada- debieron seguir integrando la categoría de infracciones administrativas aunque, claro está, eso forma ya parte de otro debate. En cualquier caso, como el deseo del legislador ha sido otro, no queda si no esperar a que las medidas reeducadoras que se están adoptando sirvan, en primera instancia, para evitar accidentes de tráfico y, a más largo plazo, lleguen a hacer innecesario el recurso al Derecho penal.

\section{Referencias}

[1] Sobre la misma puede verse BLAY GIL, E., La pena de trabajo en beneficio de la comunidad. Bellaterra, 2006; BRANDARIZ GARCÍA, J. A., La sanción penal de trabajos en beneficio de la comunidad. Ed. Tirant lo Blanch, 2009; y TORRES ROSELL, N., $L a$ pena de trabajos en beneficio de la comunidad. Reformas legales y problemas de aplicación. Ed. Tirant lo Blanch, 2006.

[2] Un comentario de la misma puede verse, entre otros, en ALONSO RIMÓ, A., "El delito de negativa a someterse a las pruebas de detección de alcohol o drogas tóxicas desde la perspectiva de la reforma penal de 2007", en Seguridad Vial. V. V. A. A. Coord. por C. VIDALES RODRÍGUEZ y A. MERA REDONDO, Ed. Tirant lo Blanch, 2008, p. 293 a 324; CARBONELL MATEU, J. C., "La ley orgánica de reforma del Código Penal en materia de seguridad vial: un comentario de urgencia", en La adecuación del Derecho penal español al ordenamiento de la Unión Europea: la política criminal europea. V. V. A. A., Coord. F. J. ALVÁREZ GARCÍA, A. MANJÓN-CABEZA OLMEDA y A. VENTURA PÜSCHEL. Ed. Tirant lo Blanch, 2009, p. 633 a 650; del mismo, "La reforma del tratamiento penal de la Seguridad Vial", en Derecho Penal y Seguridad Vial, V. V. A. A., Ed. Aranzadi, 2007, p. 57 a 74; DE VICENTE MARTÍNEZ, R., "La reforma penal en curso en materia de siniestralidad vial", en Derecho Penal y Seguridad Vial. Estudios de Derecho Judicial. Consejo General del Poder Judicial. Madrid,
2007; GARCÍA ALBERO, R., "La nueva política criminal de la seguridad vial", en Revista Electrónica de Ciencia Penal y Criminología, 09-11 (2007), disponible en http://criminet.ugr.es/cecpc; GONZÁLEZ CUSSAC, J. L., y VIDALES RODRÍGUEZ, C., "Los nuevos delitos contra la seguridad vial", en Seguridad Vial. V. V. A. A. Coord. por C. VIDALES RODRÍGUEZ y A. MERA REDONDO, Ed. Tirant lo Blanch, 2008, p. 193 a 225; de los mismos, "La reforma del Código penal en materia de seguridad vial", en Revista Xuridica Galega, no 55, 2007, p. 43 a 60; LUZÓN PEÑA, D. M., "Posibles reformas de los delitos de circulación", en Derecho Penal y Seguridad Vial, V. V. A. A., Ed. Aranzadi, 2007, p. 31 a 36; MORENO ALCÁZAR, M. A., "La criminalización de las conducciones temerarias en el marco de la nueva reforma penal en materia de seguridad vial", en Seguridad Vial. V. V. A. A. Coord. por C. VIDALES RODRÍGUEZ y A. MERA REDONDO, Ed. Tirant lo Blanch, 2008, p. 227 a 291; SOLA RECHE, E., "Los viejos problemas de los nuevos delitos contra la seguridad vial", en Revista General de Derecho penal, $\mathrm{n}^{\circ} 10,2008$. V. V. A. A. Coord. por S. MIR PUIG, M. CORCOY BIDASOLO y S. CARDENAL MONTRAVETA, Seguridad vial y derecho penal: análisis de la LO 15/2007, que modifica el Código penal en materia de seguridad vial. Ed. Tirant lo Blanch, 2008.

[3] A tenor de lo dispuesto en el precepto citado, pueden sustituirse las penas de prisión que no excedan de un 
año -y, en supuestos excepcionales, de dos- por multa o trabajo en beneficio de la comunidad. Para ello, los jueces o tribunales habrán de atender a las circunstancias personales del reo, la naturaleza del hecho, su conducta $\mathrm{y}$, en particular, el esfuerzo realizado para reparar el daño quedando excluidos, en todo caso, los supuestos de habitualidad.

[4] Memoria de la Fiscalía General del Estado, 2008, p. 699.

[5] Datos obtenidos de la Memoria de la Fiscalía General del Estado, 2009, p. 823 y 824.

[6] Memoria de la Fiscalía General del Estado, 2010, p. 967.

[7] Este plazo que, originariamente estaba previsto para un máximo de 30 jornadas, se ha ampliado, mediante la adenda a la Instrucción 2/2010, de fecha 16 de junio de 2010 .

[8] Para ampliar esta información, puede consultarse la Instrucción I-2/2010 de la Dirección General de Instituciones Penitenciarias, de 10 de marzo de 2010, disponible en http://www.institucionpenitenciaria.es/web/export/sites/default/datos/descargables/ instruccionesCirculares/I_2-2010.pdf.
[9] Memoria Fiscalía General del Estado, 2010, p. 966.

[10] Disponible en http://www.institucionpenitenciaria.es/ web/export/sites/default/datos/descargables/publicaciones/Trxfico_Programa_Editorial_2010.pdf.

[11] Al respecto, piénsese que el curso de recuperación del permiso o licencia de conducción, cuya realización y posterior superación con aprovechamiento, que es la modalidad prevista, además de para los casos de pérdida total de puntos, para quien cumple una condena privativa del derecho a conducir, tiene establecida una duración de veinticuatro horas; por tanto, escasamente superior a la fase formativa de que consta el Taller de actividades en materia de seguridad vial. Sobre la distribución de esas horas, así como los concretos contenidos a impartir, puede verse los artículos $5^{\circ}$ y $8^{\circ}$, respectivamente de la Orden INT/2596/2005, de 28 de julio, por la que se regulan los cursos de sensibilización y reeducación vial para los titulares de un permiso o licencia de conducción.

[12] En este sentido, puede verse, TORRES ROSELL, N., "La pena de localización permanente y la pena de trabajos en beneficio de la comunidad, arts. 37, 49 y 88 CP”, en La Reforma Penal de 2010: Análisis y Comentarios. Dir. G. QUINTERO OLIVARES, 2010, p. 95.

\section{Bibliografía}

Blay Gil, E.: La pena de trabajo en beneficio de la comunidad. Universitat Autònoma de Barcelona. Bellaterra, 2006.

Blandariz García, J.A.: La sanción penal de trabajos en beneficio de la comunidad. Ed. Tirant lo Blanch, Valencia, 2009.

Carbonell Mateu, J. C.: "La ley orgánica de reforma del Código Penal en materia de seguridad vial: un comentario de urgencia”, en La adecuación del Derecho penal español al ordenamiento de la Unión Europea: la política criminal europea. V. V. A. A., Coord. F. J. ALVÁREZ GARCÍA, A. MANJÓN-CABEZA OLMEDA y A. VENTURA PÜSCHEL. Ed. Tirant lo Blanch. Valencia, 2009, p. 633 a 650.

Cervera Salvador, S.: "La ejecución de la pena de trabajos en beneficio de la comunidad. Análisis del RD 1849/2009, de 4 de diciembre", en Revista Vasca de Derecho Procesal y Arbitraje. Vol. 22, n 3, 2010, p. 631 a 644. .
Dolz lago, M.J.: "Los programas de educación vial ante los delitos contra la seguridad del tráfico: una alternativa eficaz", en Actualidad Penal, no 12, 1998, p. 257 a 275.

García Albero, R.: "La nueva política criminal de la seguridad vial”, en Revista Electrónica de Ciencia Penal y Criminología, 09-11 (2007), http://criminet.ugr.es/cecpc

González Cussac, J.L. y Vidales Rodríguez, C.: "La reforma del Código penal en materia de seguridad vial", en Revista Xuridica Galega, n 55, 2007, p. 43 a 60

De Vicente Martínez, R.: “"'La reforma penal en curso en materia de siniestralidad vial", en Derecho penal y Seguridad Vial. Estudios de Derecho Judicial. Consejo General del Poder Judicial. Madrid, 2007.

Magro Servet, V.: "La reeducación obligatoria y el cumplimiento de la pena de trabajos en beneficio de la comunidad en la seguridad vial", en Tráfico y Seguridad Vial, $n^{\circ} 118,2008$, p. 5 a 12; "El Real Decreto 1849/2009, de 4 de diciembre y la introducción de la reeducación en la 
seguridad vial para cumplir la pena de trabajos en beneficio de la comunidad", en Tráfico y Seguridad Vial, n 34, 2010, p. 5 a 14.

Tamarit Sumalla, J.M., y Luque Reina, M. E.: Automóviles, delitos y penas. Estudio de la criminalidad y de las sanciones penales relacionadas con los vehículos de motor. Ed. Tirant lo Blanch. Valencia, 2007.

Torres Rosell, N.: La pena de trabajos en beneficio de la comunidad. Reformas legales y problemas de aplicación. Ed. Tirant lo Blanch. Valencia, 2006; "La pena de localización permanente y la pena de trabajos en beneficio de la comunidad, arts. 37, 49 y 88 CP”, en La Reforma Penal de 2010: Análisis y Comentarios. Dir. G. QUIN-
TERO OLIVARES, Ed. Aranzadi, Cizur Menor, 2010, p. 91 a 97.

Vidales Rodríguez, C.: "Trabajos en beneficio de la comunidad", en Comentarios a la reforma penal de 2010. Ed. Tirant lo Blanch, 2010, p. 107 a 110.

V. V. A. A., Derecho penal y Seguridad Vial. Ed. Aranzadi. Cizur Menor, 2007.

V. V. A. A. Coord. por S. MIR PUIG, M. CORCOY BIDASOLO y S. CARDENAL MONTRAVETA, Seguridad vial y derecho penal: análisis de la LO 15/2007, que modifica el Código penal en materia de seguridad vial. Ed. Tirant lo Blanch, 2008.

V. V. A. A. Coord. por C. VIDALES RODRÍGUEZ y A. MERA REDONDO, Seguridad Vial. Ed. Tirant lo Blanch. Valencia, 2008. 